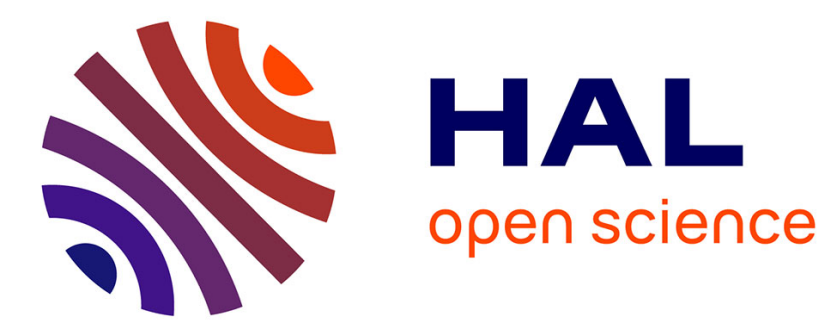

\title{
Flapping instability of a liquid jet
}

Antoine Delon, Alain H. Cartellier, Jean-Philippe Matas

\section{To cite this version:}

Antoine Delon, Alain H. Cartellier, Jean-Philippe Matas. Flapping instability of a liquid jet. Physical Review Fluids, 2018, 3 (4), 10.1103/PhysRevFluids.3.043901 . hal-01761377

\section{HAL Id: hal-01761377 \\ https://hal.science/hal-01761377}

Submitted on 11 Apr 2018

HAL is a multi-disciplinary open access archive for the deposit and dissemination of scientific research documents, whether they are published or not. The documents may come from teaching and research institutions in France or abroad, or from public or private research centers.
L'archive ouverte pluridisciplinaire HAL, est destinée au dépôt et à la diffusion de documents scientifiques de niveau recherche, publiés ou non, émanant des établissements d'enseignement et de recherche français ou étrangers, des laboratoires publics ou privés. 


\title{
Flapping instability of a liquid jet
}

\author{
Antoine Delon and Alain Cartellier* \\ Univ. Grenoble Alpes, CNRS, Grenoble INP, LEGI, F-38000 Grenoble, France \\ Jean-Philippe Matas \\ Laboratoire de Mécanique des Fluides et d'Acoustique, Ecole Centrale de Lyon, \\ CNRS, Université Claude Bernard Lyon 1, INSA Lyon, F-69134 Ecully, France
}

(Dated: April 11, 2018)

\begin{abstract}
We measure experimentally the frequency of the large-scale instability developing on a liquid jet incompletely atomized by a parallel fast gas stream. We demonstrate that this "flapping instability" can be triggered by different mechanisms: in a first regime it is synchronized with the shear instability developing upstream, provided the wavelength of this shear instability is larger than the liquid jet diameter $H_{L}$. When the shear instability exhibits wavelengths shorter than $H_{L}$, a second regime is observed where the flapping instability becomes independent of the gas stream velocity. This second regime is characterized by a constant Strouhal number, provided the Froude number of the jet is correctly taken into account.
\end{abstract}

\section{INTRODUCTION}

The destabilization of liquid jets is at the center of many industrial processes where the aim is to form a spray. In the case of assisted atomization, a liquid jet is broken into droplets with the help of a parallel annular gas flow [1]. It has been demonstrated that in this configuration the formation of drops results from a series of processes: a shear instability between the slow liquid and high speed gas stream leads to the formation of waves, the crest of these waves is accelerated by the wind and destabilized into ligaments and then ultimately broken into droplets [2-5]. When the jet is incompletely atomized, large liquid lumps remain close to the axis. It was pointed out by Farago \& Chigier (1992) [6] that for certain conditions the break-up of the liquid jet was non axisymmetric (see figure 1), with a wavelenth very large compared to the jet diameter. As pointed at in Eroglu et al (1991) [7], the motion of the liquid jet exhibits in this regime a flapping motion. The same instability, either termed flapping or helicoidal instability, is observed in subsequent studies involving the atomization of a liquid jet, but is not studied as such [2, 5, 8-12]. Juniper \& Candel (2003) [10], who observe it when liquid injection is recessed, suggest it is a wake instability. All the above experiments were carried out with water jets, but the instability has also been observed in the case of cryogenic fluids, in conditions close to those of rocket engines [13], see figure 1 bottom.

The aim of the present paper is to clarify the nature of this instability in a wide range of geometries, gas and liquid velocities. We will present measurements of the frequency of the instability, and compare those to the predictions of a linear stability analysis. We will show that in a first regime the shear instability which develops upstream controls the flapping instability, while for other

\footnotetext{
* Institute of Engineering Univ. Grenoble Alpes
}

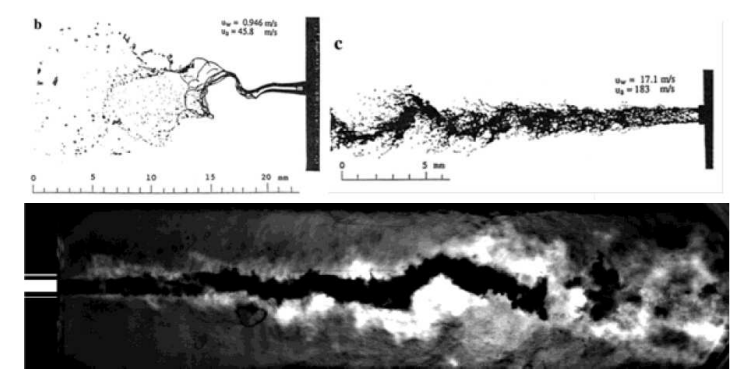

FIG. 1. Top: large scale jet oscillations observed by Farago \& Chigier (1992) [6]. Bottom: lateral oscillations observed by Locke et al 2010 on cryogenic fluids [13]

conditions a new regime where the flapping instability is independent of the gas stream velocity takes place. Section II introduces the experimental set-up and results. Section III presents the stability analysis, and the comparison of its results to experimental data. Section IV discusses the results obtained in the gas-independent second regime.

\section{EXPERIMENTAL RESULTS}

\section{A. Experimental setup}

The injector used in this work is represented on Figure 2. It is composed of two coaxial steel cylinders: the liquid goes through the inner cylinder, and the gas is injected in the annular region between both cylinders. Their length $(\approx 1 \mathrm{~m})$ is long enough to ensure fully developed flow conditions for both phases. The liquid injector is formed of two tubes. The bottom one can be changed in order to modify the exit diameter $H_{L}$ : the latter has been varied from 5 to $20 \mathrm{~mm}$. Water is supplied by an overflowing tank, in order to ensure a stable flow rate troughout measurements. For the larger liquid diameters $\left(H_{L}=15 \mathrm{~mm}\right.$ and $\left.20 \mathrm{~mm}\right)$, liquid velocities were 


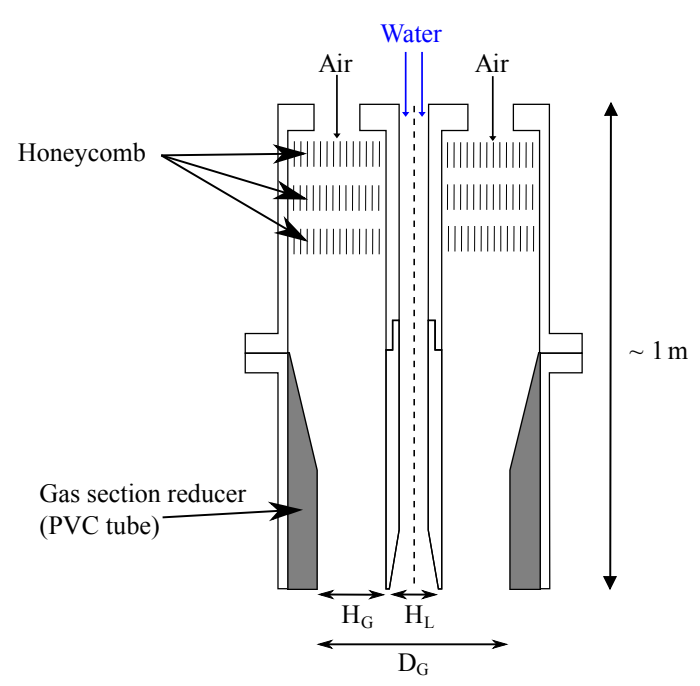

FIG. 2. Coaxial injector used in the present work.

limited below $1 \mathrm{~m} / \mathrm{s}$ with this feeding system : a $100 \mathrm{l}$ bladder pressure tank was therefore used to reach higher jet velocities. The liquid flow rate is measured with an Oval flowmeter LSF445 (range 8 to 100 liters per hour, uncertainty of $1 \%$ of measured value). The mean liquid velocity $U_{L}$ is deduced from this measurement. Liquid velocities were varied in the range $[0.17-1.4] \mathrm{m} / \mathrm{s}$.

The outer cylinder is also composed of two tubes: the top one is equipped with a damping chamber (honeycomb) and the bottom one can be changed to modify the gas exit diameter $D_{G}$, and hence the gas stream thickness $H_{G}$. This thickness $H_{G}$ spans the range 1.8 to 24 $\mathrm{mm}$ in the present experiments. The relatively long tube (length/width ratio in the range 41-500 depending on liquid diameter) allows the development of thick boundary layers. Clean air at room temperature delivered from a compressor feeds the gas stream. The maximum air velocity $U_{G 0}$ is measured at the exit of the gas channel, at the center of the gas ring $\left(H_{G} / 2\right)$, with a Pitot tube and a differential pressure sensor TSI DpCalc (uncertainty $1.5 \%$ of the read value). Mean velocity $U_{G}$ can be deduced from the flow rate, measured with a mass flow meter Brooks SLA 5860. Gas velocities have been varied in the range 10 to $140 \mathrm{~m} / \mathrm{s}$.

Velocity profiles in the gas stream are measured in the exit section with a $5 \mu \mathrm{m}$ hot wire and a DISA anemometer, at a distance of $0.2 \mathrm{~mm}$ below the injector lip. Examples of velocity profiles for one injector are given in figure 3. Position zero corresponds to the outer edge of the separator lip between the liquid and gas injectors. We can deduce from these profiles the vorticity thickness $\delta_{G}$ : $\delta_{G}=\Delta U /\left.\frac{d U}{d r}\right|_{\max }$ which is measured for each geometry as a function of mean gas velocity $U_{G}$. The hot wire measurements also give access to velocity fluctuations and turbulence intensity profiles (not shown). These profiles indicate that for all geometries and $U_{G}$ in the range [15$110] \mathrm{m} / \mathrm{s}$ the turbulence intensity, defined as the ratio of the rms velocity $u_{r m s}$ to the mean velocity, is of the
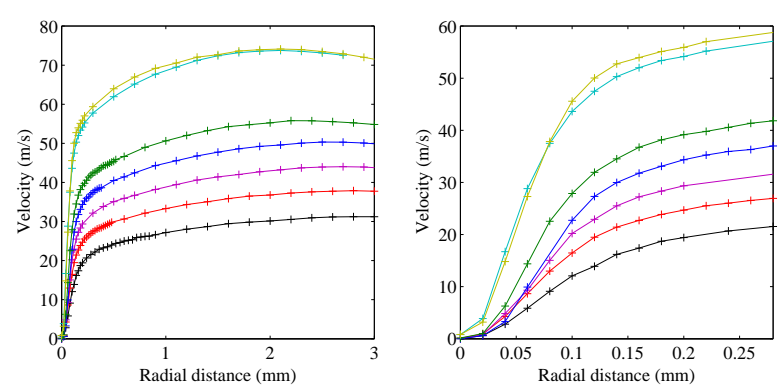

FIG. 3. Left: Velocity profiles in the gas channel for the $H_{L}=5 \mathrm{~mm}$ and $H_{G}=5 \mathrm{~mm}$ geometry. Origin of the radial distance is taken at the outer edge of the splitter lip. Right: zoom showing the boundary layer is spatially resolved.

order of $10 \%$ close to the boundary layer, down to $4 \%$ at the center of the gas channel. Turbulence intensity is larger for the $H_{L}=5 \mathrm{~mm}-H_{G}=12.5 \mathrm{~mm}$ geometry, around $20 \%$ at the center of the channel, probably due to the formation of recirculations within the gas channel for this particular injector. The lip of the splitter tube that separates the gas from the liquid has a thickness of $e=0.2 \mathrm{~mm}$ for all injectors. For all the experimental conditions considered here, this lip thickness remains smaller than the gas vorticity thickness $\left(\right.$ ratios $\delta_{G} / e$ between 1 and 3 for all conditions).

Pictures of the liquid jet are taken with a Vision Research Miro M310 high-speed camera equipped with a TAMRON $90 \mathrm{~mm}$ objective set at full aperture. The spatial resolution is about $0.2 \mathrm{~mm}$ per pixel. The exposure time was set to $90 \mu \mathrm{s}$. A typical example of collected images is shown in figure 4: it illustrates the flapping motion characterized by lateral displacements larger than the liquid jet injection radius. The image processing of such pictures will be described in the following subsection.

\section{B. Flapping frequency}

In order to measure the frequency of the flapping instability, raw shadowgraph images of the liquid jet are processed in the following manner: Background elimination is performed, and a median filter is applied whose size is chosen so as to eliminate drops and ligament structures. Its size must therefore be adjusted according to the camera resolution and to the sizes of the structures to be eliminated. In the present experiments, the filter size ranges from 2 pixels to 40 pixels, in order to cut off objects with a size smaller than $1 \times 1$ pixel up to $20 \times 20$ pixels respectively. A horizontal gray level profile is then extracted for various downstream positions of the jet center.

On each profile, the jet center position $x_{C}$ is computed from the intensity profiles $I(x)$ as $x_{C}=$ $(\Sigma x I(x)) /(\Sigma I(x))$, where $x$ is the distance to the axis of symmetry of the injector. For each flow condition, 

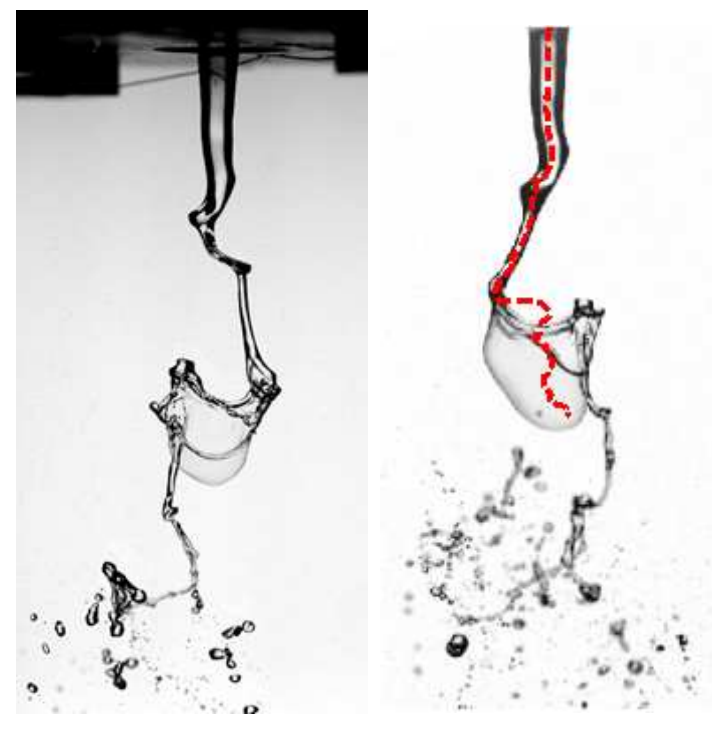

FIG. 4. Left: Typical image of a flapping jet for $H_{L}=5 \mathrm{~mm}$, $H_{G}=5 \mathrm{~mm}, U_{L}=0.28 \mathrm{~m} / \mathrm{s}, U_{G}=19.5 \mathrm{~m} / \mathrm{s}$. Right: Raw image and detected jet center shown in red dashed line.

the data set consists in a time series of 5000 pictures taken at a sampling frequency of $1 \mathrm{kHz}$. For each timeseries, the computed liquid jet center is superimposed onto the original pictures on a movie, in order to visually check the correct functioning of the algorithm and also the good tuning of the filter length. A typical output of this data processing is shown in figure 4 right. The jet center determination is quite accurate in regions where the liquid jet is close to a cylinder-like shape. In other regions, in particular whenever bag formation occurs, the jet center is somewhat ill-defined, but the image processing provides continuous information, and the resulting spectra never exhibit spurious discontinuities because of jet shape. A typical example of the spatial evolution of the flapping frequency spectrum is given in figure 5: the peak frequency remains the same whatever the downstream distance. The intensity of the peak increases with downstream distance due to the increase in flapping amplitude.

Flapping frequency measurements are plotted in figure 6 against gas velocity $U_{G 0}$ (measured at the center of the gas channel exit) for various geometries and $U_{L}$. Given the large number of parameters in this problem, and in particular of length/velocity scales, we present first the dimensional data as such: suggestions to properly nondimensionalize this data will be introduced in the course of the discussion in sections III and IV. Two groups of data can be distinguished on figure 6 :

- A first group where the flapping frequency increases with gas velocity, which we label $G_{1}$. This includes all the data for the $H_{L}=5 \mathrm{~mm}$ injector, and the series for $H_{L}=20 \mathrm{~mm}-H_{G}=24 \mathrm{~mm}$.

- A second group where the flapping frequency is almost independent of gas velocity, named $G_{2}$. This

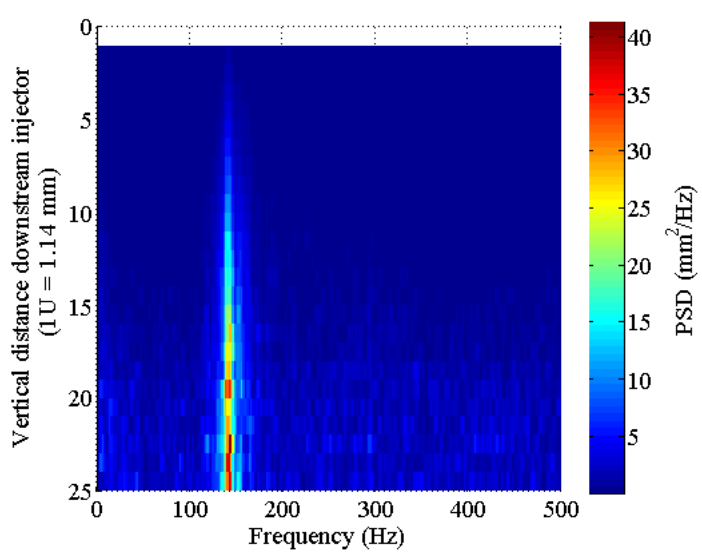

FIG. 5. Spectrum of flapping frequency as a function of downstream distance from nozzle for $H_{L}=5 \mathrm{~mm}, H_{G}=5 \mathrm{~mm}$, $U_{L}=0.28 \mathrm{~m} / \mathrm{s}$ and $U_{G}=45.5 \mathrm{~m} / \mathrm{s}$.

group includes the data for all $H_{L}=15 \mathrm{~mm}$ and $H_{L}=20 \mathrm{~mm}$ geometries, except the $H_{L}=20 \mathrm{~mm}-$ $H_{G}=24 \mathrm{~mm}$ series.

Regime $G_{1}$ where the flapping frequency monotonically increases with gas velocity is reminiscent of the regime observed in sheet atomization (see among others Lozano \& al 2005 [14], Arai \& Hashimoto [15]), where the fast gas stream leads to a similar flapping motion on a length scale large compared to the liquid sheet thickness. However, the fact that we observe two distinct behaviors, including one for which flapping frequency is independent of gas velocity, is new and has never been described in the literature neither for jets nor for sheets. Note that Lozano \& al (2005) [14] varied the liquid sheet thickness by a factor as large as 10 , but do not observe regime $G_{2}$.

We now discuss the sensitivity of the flapping frequency to liquid velocity. We plot on figure 7 (respectively 8) the variations of frequency as a function of $U_{L}$ for conditions corresponding to the $G_{1}$ (respectively $G_{2}$ ) series. Flapping frequency increases with liquid velocity irrespective of the regime. Yet, the increase of flapping frequency with liquid velocity is not as regular for the $G_{2}$ regime compared to the $G_{1}$ regime, in particular at low $U_{L}$. We attribute this to the acceleration due to gravity, which induces strong spatial variations at low $U_{L}$ : in particular, gravity significantly increases liquid velocity and reduces the radius of the liquid jet over distances short compared with $H_{L}$ in the $H_{L}=20 \mathrm{~mm}$ case. Put differently, this corresponds to the fact that the low velocity data of figure 8 corresponds to a Froude number $\mathrm{Fr}=U_{L} / \sqrt{g H_{L}}$ of order one, while the data at larger $U_{L}$ and in particular for the $H_{L}=15 \mathrm{~mm}$ series, corresponds to $\mathrm{Fr}$ significantly larger than one. The influence of liquid velocity on frequency in regime $G_{2}$ can also be noticed on figure $6 \mathrm{~b}$ ), by comparing the two series of symbols $\triangle$ and $\boldsymbol{\Delta}$, which correspond respectively to $U_{L}=0.08 \mathrm{~m} / \mathrm{s}$ and $U_{L}=0.25 \mathrm{~m} / \mathrm{s}$, both for $H_{L}=15 \mathrm{~mm}$ and $H_{G}=4.3 \mathrm{~mm}$. The pressure bladder 

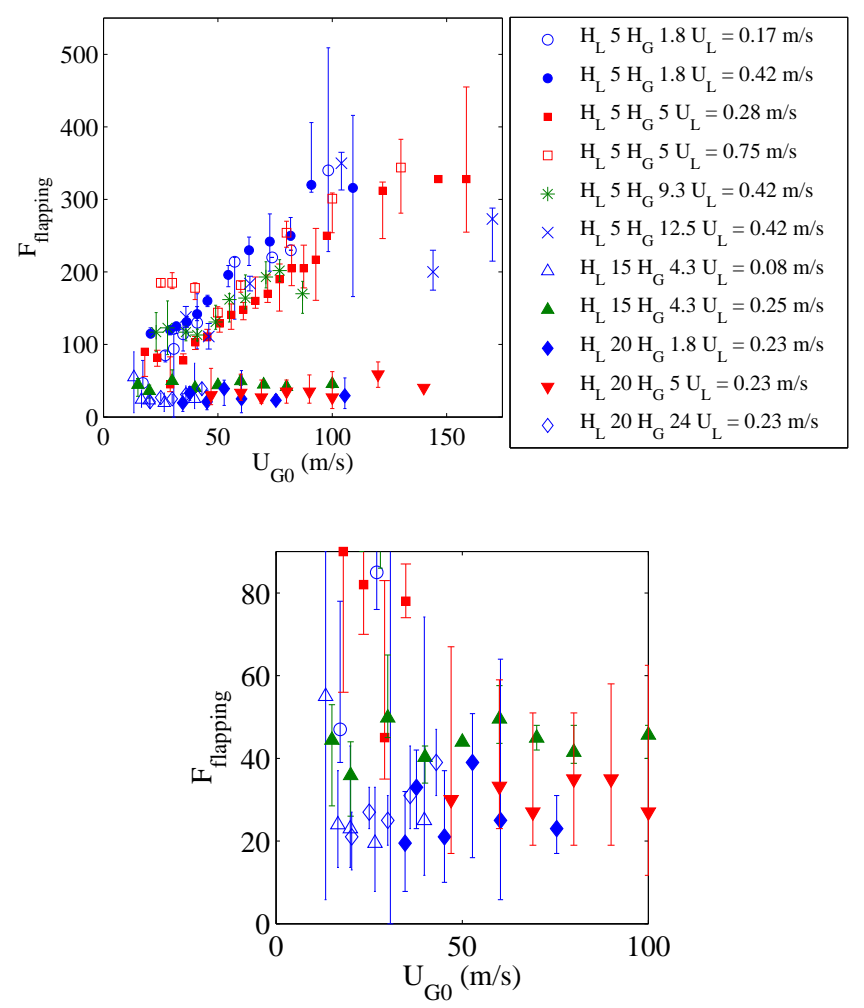

FIG. 6. a) Variation of flapping instability frequency with gas velocity for different injector geometries and various liquid velocities. Error bars represent the spectrum peak width at mid-height. Values of $H_{G}$ and $H_{L}$ in the legend are in $\mathrm{mm}$. b) Same data, zoom on low frequency data.

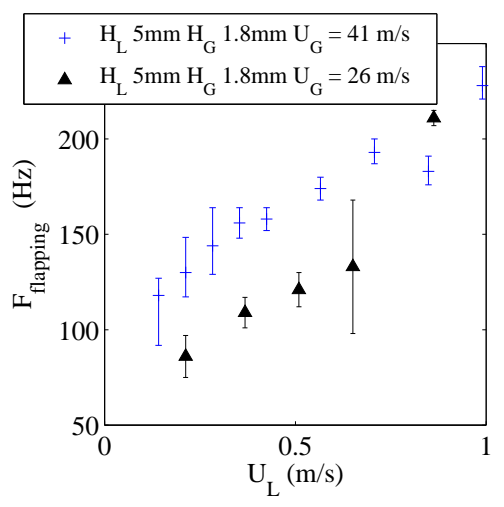

FIG. 7. Frequency of the flapping instability as a function of liquid velocity $U_{L}$, for the $G_{1}$ series.

tank (see section II A) was needed to reach the conditions of figure 8: even with this device, it was not possible due to limitations in the maximum pressure allowable in our set-up to reach higher $U_{L}$ than those of figure 8 for the large $H_{L}$ geometries. Finally, note that a flapping frequency increasing with liquid velocity is also observed by Lozano et al. (2005) [14] in sheet atomization for liquid velocities up to $1-1.5 \mathrm{~m} / \mathrm{s}$. At higher liquid velocities,

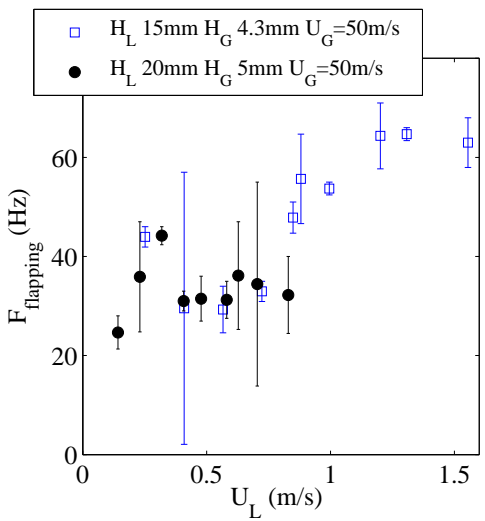

FIG. 8. Frequency of the flapping instability as a function of liquid velocity $U_{L}$ for $G_{2}$ series.

they observe a variation of frequency with liquid velocity which is no longer monotonic.

Diverse proposals, mostly empirical, have been made in the literature regarding the scaling of the flapping frequency with $U_{G}$ for the case of liquid sheets. Different Strouhal numbers built on various length scales (namely $H_{L}, H_{G}, \delta_{G}$ or some combination of these) have been considered by authors. We have tested the propositions from Arai \& Hashimoto (1985) [15], Lozano et al 2001 [16], Couderc 2007 [17] and Odier et al 2014 [18], but they all fail to collapse the data of figure 6 . In particular, none of the above propositions provides a satisfactory dependence of frequency on the liquid thickness $H_{L}$.

The data of figure 6 suggest that two distinct mechanisms can pilot the flapping instability. We will first focus on the $G_{1}$ series, for which frequency increases with both $U_{G}$ and $U_{L}$. This behaviour is similar to that of shear instabilities identified in the context of liquid jet atomization $[3,19]$. We will therefore in the next subsection characterize the shear instability occurring upstream of the flapping instability.

\section{Shear instability frequency}

The shear instability occurs close to the nozzle exit, while the flapping instability occurs (and has been characterized) farther downstream. In order to detect a possible connection between both instabilities, we need to identify a way to characterize them independently. We choose to determine the frequency of the shear instability via the variations of the local jet radius $R(z, t)$, measured at a given downstream distance $z$. We apply the following image processing under MATLAB: Raw pictures obtained with shadowgraphy and high-speed imaging of the jet are background-removed, thresholded with Otsu's method, and segmented (bwlabel function). This series of operations provides the area connected to the injector exit, corresponding to the liquid tongue. For each downstream distance $z$, we extract the left/right borders of the 


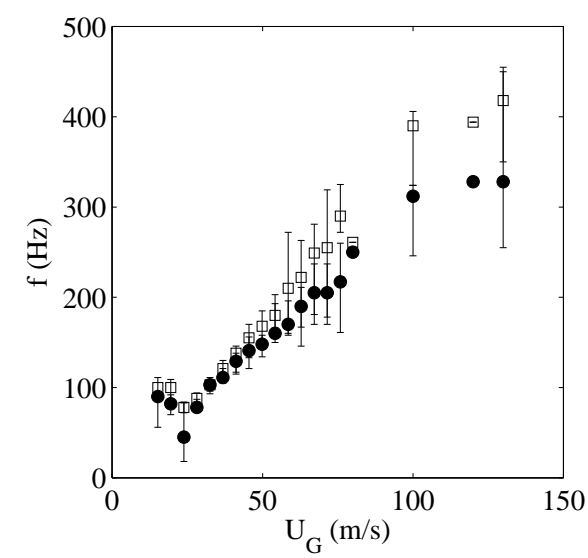

FIG. 9. Flapping $\bullet$ and shear wave $\square$ frequency as a function of air velocity. Experimental conditions: $H_{L}=5 \mathrm{~mm}, H_{G}=$ $5 \mathrm{~mm}, U_{L}=0.28 \mathrm{~m} / \mathrm{s}$.

jet, and compute the local jet radius $R(z, t)$, given by the distance between opposite borders at a given downstream location $z$. These signals were extracted at regular positions between the nozzle exit and a downstream distance of $3 H_{L}$. Spectra of the local jet radius are obtained with Fast Fourier Transform. Shannon criterion is always fulfilled, and the frequency resolution is $0.24 \mathrm{~Hz}$.

In figure 9 , we plot the frequency of the shear waves measured from the spectrum of $R(z, t)$, along with the flapping frequency (measured farther downstream following the method described in section IIB) for the $H_{G}=5 \mathrm{~mm}$ and $H_{L}=5 \mathrm{~mm}$ injector. The data show that both frequencies are close: the flapping frequency closely follows the frequency of the shear waves formed near the nozzle. This closeness suggests a relation between both instabilities, but at the same time the fact that the (downstream) flapping instability frequency is systematically smaller than the (upstream) shear instability frequency suggests that the instabilities are actually not synchronous. The difference between both frequencies is illustrated on figure 10 for the same injector and liquid velocity as figure 9 , and a fixed $U_{G}=23.8 \mathrm{~m} / \mathrm{s}$. The top spectra, obtained for several values of $z$, are that of the radius $R(t)$, and provide the shear instability frequency ; the bottom spectra, also for several $z$, are the spectra of the jet center location $x_{C}(t)$, which provide the flapping frequency introduced in section IIB, and which is measured farther downstream. The spectra in the middle are the spectra of the jet edge, and exhibit two main distinct peaks: one corresponds to symmetrical waves (top spectra) and the other one to the flapping frequency (bottom spectra). This confirms that both frequencies are actually present in the jet: the interface position is affected both by large-scale motions of the jet as well as by interfacial waves on top of it. These features are the same whatever the downstream position, down to $z=3 H_{L}$. Beyond this distance, it becomes difficult
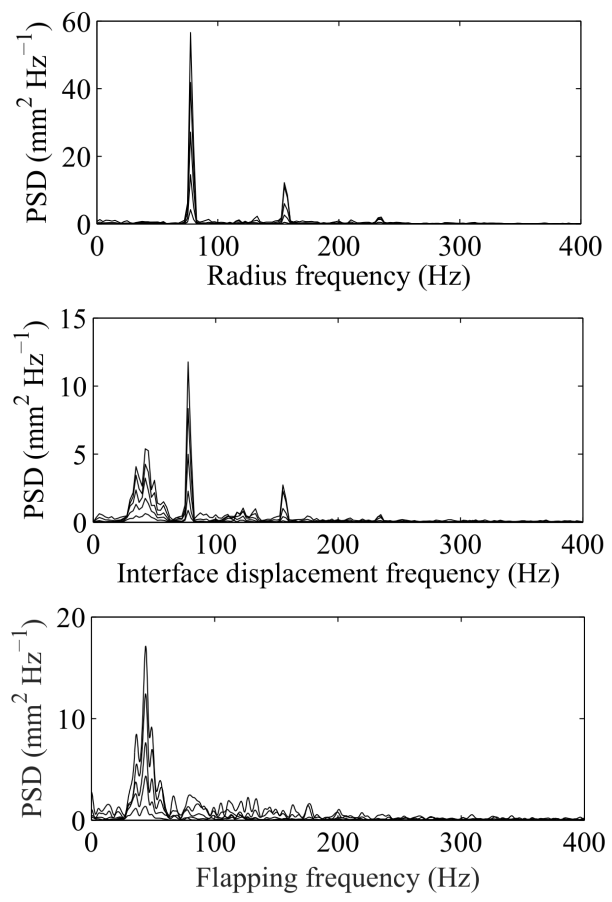

FIG. 10. Top: spectrum of jet radius for downstream distances from $z=4.1 \mathrm{~mm}$ down to $12.5 \mathrm{~mm}$, with a step of $\Delta z=2.1 \mathrm{~mm}$, showing a maximum frequency of $f=77 \mathrm{~Hz}$, and a harmonic at $154 \mathrm{~Hz}$. Middle: spectrum of jet edge at same fixed $z$. Bottom: spectrum of jet center $x_{C}$ (see section II B) from $z=6.9 \mathrm{~mm}$ down to $z=22.9 \mathrm{~mm}, \Delta z=4 \mathrm{~mm}$. The middle spectrum clearly corresponds to a sum of the top and bottom spectra. Experimental conditions: $H_{L}=5 \mathrm{~mm}$, $H_{G}=5 \mathrm{~mm}, U_{L}=0.28 \mathrm{~m} / \mathrm{s}$ and $U_{G}=23.8 \mathrm{~m} / \mathrm{s}$.

to isolate a maximum frequency in the spectra of $R(z, t)$. A very interesting feature is that the flapping frequency is not present in the jet radius spectrum (top graph): this implies that flapping waves do not result from the amplification of symmetric perturbations. However, the close observation of shear waves shows that some of these waves are not symmetric: we postulate that the flapping results from the amplification of such non axisymmetric waves. This is supported by figure 11, which gives an example of an asymmetric wave and of its evolution in time: oblique waves continuously evolve to ultimately form large-scale structures characteristic of the flapping instability.

Previous linear stability analyses on air/water jet atomization were focused on the search for $2 \mathrm{D}$ or varicose perturbations $[3,20]$. In the next section, we discuss the stability of non axisymmetric perturbations in order to assess their possible role in triggering the flapping instability. 


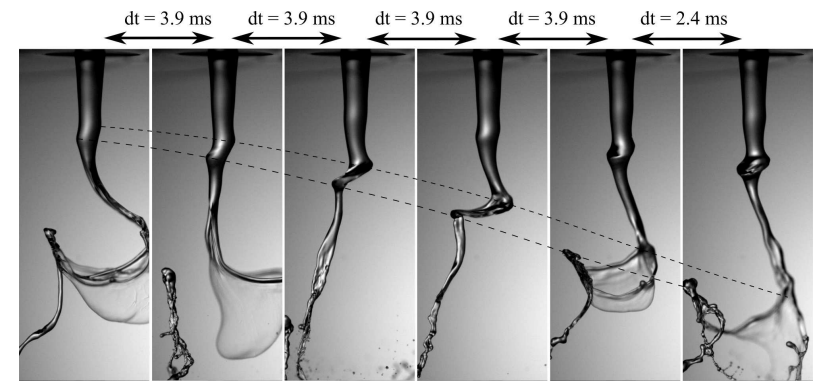

FIG. 11. Time evolution of an oblique shear instability wave, for $H_{L}=5 \mathrm{~mm}, H_{G}=5 \mathrm{~mm}, U_{L}=0.28 \mathrm{~m} / \mathrm{s}$ and $U_{G}=$ $22 \mathrm{~m} / \mathrm{s}$

\section{STABILITY ANALYSIS}

\section{A. Method}

Stability analysis of the air/water mixing layer configuration for large velocity ratios and in the case of a finite gas vorticity thickness $\delta_{G}$ was carried out by Marmottant \& Villermaux (2004) in the frame of an inviscid temporal stability analysis [3]. This simple approach managed to capture the scaling of shear waves frequency with $U_{G}$ at large gas velocities, but underestimated frequencies by a factor three. It was later shown that inclusion of viscosity in the analysis led to a more complex pattern: when the instability is convective (typically for larger liquid velocities) its mechanism is essentially viscous; for most conditions relevant to experiments it is, however, absolute [21-23]. Two distinct mechanisms can cause absolute instability, either surface tension if $W e_{U i}=\rho_{L} U_{i}^{2} / \sigma k_{i}^{*}<1$, or confinement if $W e_{U i}>1$ and $M=\rho_{G} U_{G}^{2} / \rho_{L} U_{L}^{2} />1$. We have introduced in these expressions the liquid and gas densities $\rho_{L}$ and $\rho_{G}$, the interfacial velocity $U_{i}$, surface tension $\sigma$ and the spatial growth rate $k_{i}^{*}$ of the most dangerous shear mode [20]. When the instability is absolute because of a confinement branch (i.e. at large $W e_{U i}$ and $M$, which typically correspond to the larger $U_{G}$ reached in atomization experiments), the unstable perturbation is fed by Reynolds stresses, and it can be shown that the energy budget is in this particular case similar to that of the simplified inviscid approach.

Our aim in this study is to clarify the stability of non axisymmetric modes, and compare their frequency to the frequency of the varicose mode. The interface perturbation can be developed on normal modes perturbations of the form $\left.\eta=\tilde{\eta} e^{i(k z-\omega t+n \theta}\right)$, where $k$ is the wavenumber, $\omega$ the pulsation, $\theta$ the azimuthal angle in a cylindrical frame centered on the jet axis and $n$ the number of the corresponding Fourier mode. Number $n=0$ corresponds to varicose perturbations, while $n=1$ corresponds to a helical perturbation. Non-axisymmetric perturbations with a plane of symmetry, such as illustrated on figure 11, can be reconstructed with the superposition of two $n=1$ and $n=-1$ modes. However, the inclusion of $n \neq 0$ modes in the viscous stability analysis makes it arduous to decouple the equations for velocity/pressure perturbations. Our strategy is to take advantage of the result mentioned above: we will focus on conditions for which the viscous stability analysis predicts that the mechanism is inviscid for the $n=0$ mode, and then look at the stability and frequency of the $n=1$ mode within the assumptions of the simplified inviscid approach.

\section{B. Stability analysis for helical modes}

We consider the experimental shear instability data of figure 9 (symbol $\square$ ), for which $H_{G}=5 \mathrm{~mm} H_{L}=5 \mathrm{~mm}$ and $U_{L}=0.28 \mathrm{~m} / \mathrm{s}$. We focus on the point $U_{G}=45 \mathrm{~m} / \mathrm{s}$, for which the frequency is measured at $f=155 \mathrm{~Hz}$. We first carry out for these conditions the spatio-temporal viscous stability analysis already introduced in Matas et al (2018) [20] for similar atomization conditions. The details of this viscous analysis are presented in the appendix: the aim is to determine if the main destabilizing mechanism is actually viscous, or on the contrary inviscid for these particular conditions.

For the chosen conditions, we find that the shear branch pinches with a confinement branch centered around $k_{i}=1300 \mathrm{~m}^{-1}$, corresponding to a confinement length $L \approx 5 \mathrm{~mm}$, see figure $12 \mathrm{a}$. This pinching occurs for a positive $\omega_{i}=\operatorname{Im}(\omega)=300 \mathrm{~s}^{-1}$ : the instability is absolute. The frequency at this pinch point is $165 \mathrm{~Hz}$, in relatively good agreement with the experimental value. The physics behind this absolute instability, which is similar to the resonance of a vibrated string, is basically that the cross stream "wavelength" of the shear branch, given by $k_{i}^{-1}$, matches the cross stream confinement length $L$ for the conditions of the saddle point. An energy budget, carried out following the method initially introduced by Boomkamp \& Miesen (1996) [24], indicates that $65 \%$ of the total kinetic energy rate of the corresponding eigenmode is pumped from Reynolds stresses in the fast gas stream. The contribution of the power of viscous tangential stresses at the interface, though not negligible, only amounts to $18 \%$ of the total kinetic energy rate for this case. Given that the energy budget is dominated by the inviscid contribution, we now confidently turn to the simplified inviscid stability analysis to discuss the relative stability of the $n=0$ and $n=1$ modes.

This inviscid linear stability analysis is similar to the one carried out in previous studies on the planar mixing layer geometry $[3,19]$ except that it is here carried out in cylindrical coordinates. The base flow consists in a constant velocity equal to $U_{L}$ in the liquid phase, and in an error function in the gas accounting for the vorticity thickness $\delta_{G}$. The equations in cylindrical coordinates for the radial velocity perturbation $\tilde{v}(r)$ are the same as the ones introduced in Matas et al (2013) [25], the only difference being the boundary conditions. In the present case, we set : (i) that this velocity must be zero on the jet axis, $\tilde{v}(0)=0$; (ii) that velocity and normal stress must be continuous across the liquid/gas interface at $r=H_{L} / 2$ 
; (iii) that velocity and normal stress must be continuous at a location $r=R+H_{G}+20 \delta_{G}$ far from the interface, where we connect our integrated solution to the analytical solution $v(r)=d K_{n}(r) / d r$ valid for a constant velocity profile. Here $K_{n}(r)$ is the modified Bessel function of the second kind of order $n$. As mentioned previously, though inviscid analysis captures the right physics and trends of frequency with gas and liquid velocity, it underestimates frequency by a factor three. The aim here is to look at the stability of $n=1$ modes: our strategy is to artificially reduce the vorticity thickness $\delta_{G}$, in order to match frequencies for the $n=0$ mode, and then compare what the prediction is for the helical mode in the same conditions. No deficit is included in the base flow velocity profile in order to limit the number of parameters to adjust: one could on the contrary include a finite velocity deficit, which would limit the magnitude of the $\delta_{G}$ reduction needed to match the experimental data (see [19]), but again the aim here is to limit the number of parameters within this approach.

We first carry out a spatial approach, i.e. solve the resulting dispersion relation for real $\omega$ and complex $k$. Figure $12 \mathrm{~b}$ shows the variations of the spatial growth rate $k_{i}$ as a function of frequency, for both the $n=0$ and the $n=1$ shear modes, for the point at $U_{L}=0.28 \mathrm{~m} / \mathrm{s}$ and $U_{G}=45 \mathrm{~m} / \mathrm{s}$ introduced previously. The $n=0$ mode, symbol $\bullet$, exhibits as expected a most dangerous mode corresponding to the experimental frequency for these conditions. The $n=1$ mode (symbol $\square$ ) presents an interesting feature: at lower frequencies the spatial growth rate converges to a finite value $k_{i} \approx 700 \mathrm{~m}^{-1}$. This is the signature of an absolute instability involving a confinement branch. This is verified with a spatio temporal approach, i.e. considering $\omega_{i}=\mathcal{I} m(\omega)>0$. Figure 12c shows that for $\omega_{i}=90 \mathrm{~s}^{-1}$ the same $n=1$ shear branch pinches with a confinement branch (symbol $\square$ ) lying along the $k_{i}$ axis: the $n=1$ branch in figure $12 \mathrm{~b}$ results from a branch switching between both branches when $\omega_{i}$ is decreased down to zero in the purely spatial analysis. The confinement branch involved in the pinching is located around $550 \mathrm{~m}^{-1}$, which corresponds to a confinement length $L$ of order $10 \mathrm{~mm} \sim 2 H_{L}$. This factor 2 implies that the perturbation associated with the helical mode has opposite phases on opposite sides of the liquid jet: it can have a resonance for a wavelength of $2 H_{L}$, while this cannot be observed for the varicose mode which necessarily has identical phases on opposite sides. The absolute instability takes over the convective one, and the helical mode is therefore predicted to overcome the varicose one (symbol $\bullet$ in figure $12 \mathrm{~b}$ ) for these conditions. The fact that sinuous or helical modes can be more unstable than axisymmetric modes is well known (see e.g. Batchelor \& Gill (1962) [26] who initially pointed to the instability of helical modes in monophasic jets). The idea is that for a finite radius of the liquid jet, wavy perturbations at the jet surface are expected to be enhanced if they are of opposite phase on opposite sides of the jet, as is the case in flapping sheets for example. The radius

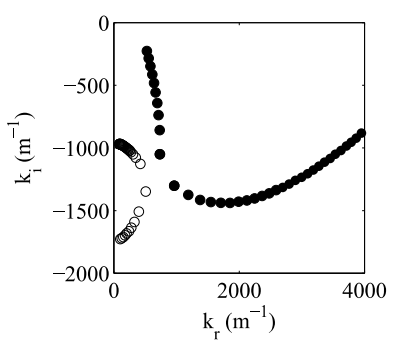

a)

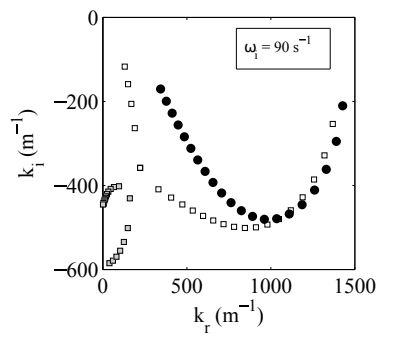

c)

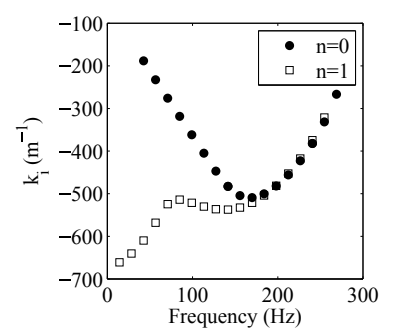

b)

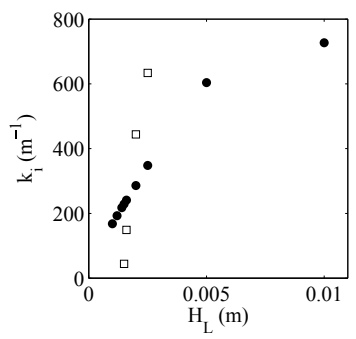

d)
FIG. 12. a) Absolute instability predicted by viscous spatiotemporal stability analysis, for $H_{L}=5 \mathrm{~mm}, H_{G}=5 \mathrm{~mm}$, $U_{L}=0.28 \mathrm{~m} / \mathrm{s}, U_{G}=45 \mathrm{~m} / \mathrm{s}, \delta_{G}=145 \mu \mathrm{m}, \delta_{L}=1 \mathrm{~mm}$ and $\delta_{d}=0.3$. Absolute growth rate is $\omega_{i}=300 \mathrm{~s}^{-1}$. Open symbols show the confinement branch, and solid symbols the shear branch. They pinch for a frequency of $165 \mathrm{~Hz}$; b) Dispersion relation predicted by spatial inviscid stability analysis for the same geometry and velocities, and $\delta_{G}=52 \mu \mathrm{m}$. c) Spatio temporal inviscid analysis for the same conditions, with $\omega_{i}=90 \mathrm{~s}^{-1}$, showing how the $n=1$ shear branch of b) (symbol $\square$ ) results from the pinching/branch switching with a confinement branch lying along the $k_{i}$ axis (symbol $\square$ ). d) Variations of the growth rate of the most dangerous mode as a function of liquid jet diameter $H_{L}$, for fixed $U_{L}=0.28 \mathrm{~m} / \mathrm{s}$ and $U_{G}=45 \mathrm{~m} / \mathrm{s}$. For $H_{L}>2.5 \mathrm{~mm}$, the instability is absolute for the $n=1$ mode (symbol $\square$ ).

of the liquid jet clearly plays an important part in enhancing the helical mode over the varicose one: we plot on figure $12 \mathrm{~d}$ the variations of the spatial growth rate of both modes as a function of $H_{L}$ (all other parameters are fixed). When the liquid jet diameter is decreased, the varicose mode becomes less unstable, and its $k_{i}$ monotonically decreases (symbol $\bullet$ ). The behaviour for the $n=1$ mode is more complex: for $H_{L}>2.5 \mathrm{~mm}$, corresponding to $\lambda<H_{L}$, the instability is absolute, with a confinement branch located at $k_{i} \approx \pi / H_{L}$. The absolute growth rate $\omega_{0 i}$ (the value of $\omega_{i}$ when the pinching occurs) reaches a maximum for $H_{L}=H_{G}=5 \mathrm{~mm}$, which is consistent with the observation of Healey (2009) [27] that this resonance mechanism is enhanced by a symmetric confinement. For $H_{L}<2.5 \mathrm{~mm}$, the instability is convective: the growth rate of its most dangerous helical mode (symbol $\square$ ) decreases rapidly when $H_{L}$ is decreased, and the helical mode eventually becomes less unstable than the varicose mode for $H_{L}<1.8 \mathrm{~mm}$. This limit corresponds to wavelengths such that $\lambda>1.5 H_{L}$. 
The inviscid analysis therefore predicts that the helical mode is more unstable than the varicose one, provided the liquid diameter is not too small. How does the frequency of this helical mode compare with that of the varicose mode? At very small liquid diameters, when the helical mode is convective, we find that the frequency of the helical mode is slightly larger than that of the varicose mode. However, when the helical mode causes an absolute instability with a confinement branch, the frequency of this mode will necessarily be smaller than the frequency of the varicose mode, since the associated saddle point is always located at low wavenumbers, hence at a frequency smaller than that of the shear branch most unstable mode: this is in agreement with the experimental observations of figure 9, where the flapping instability exhibits a frequency smaller than that of the varicose shear instability.

In order to provide an estimate for the flapping frequency, we finally come back to the viscous analysis. It has been shown in Matas et al (2018) [20] that when the viscous instability is controlled by confinement, frequency of the varicose mode at the saddle point could be estimated at moderate gas velocities by

$$
f \sim \frac{\sqrt{\frac{\rho_{G}}{\rho_{L}} \frac{\delta_{L}}{\delta_{G}}} U_{G}+U_{L}}{L}
$$

where $\delta_{L}$ is the vorticity thickness on the liquid side, and $L$ is the cross-stream length relevant to confinement: the confinement branch is located on the imaginary axis around $k_{i} \approx 2 \pi / L$. This length can a priori be either $H_{L}$ or $H_{G}$, or even a multiple of these, but there is yet no clear criterion indicating which one should be chosen. This may depend in particular on the precise shape and maximum $k_{i}$ of the shear branch for the considered liquid/gas velocities. The viscous stability analysis for the varicose mode shows that in the $H_{L}=5 \mathrm{~mm}-H_{G}=$ $1.8 \mathrm{~mm}, H_{L}=5 \mathrm{~mm}-H_{G}=9.3 \mathrm{~mm}$ and $H_{L}=5 \mathrm{~mm}-$ $H_{G}=12.3 \mathrm{~mm}$ cases the relevant confinement branch remains controlled by $L \approx H_{L}=5 \mathrm{~mm}$ independent of the value of $H_{G}$. For the series $H_{L}=20 \mathrm{~mm}-H_{G}=24 \mathrm{~mm}$, the only geometry with $H_{L}=20 \mathrm{~mm}$ in the $G_{1}$ category, this length is $L \approx 20 \mathrm{~mm}$ [20]. This suggests that the liquid diameter $H_{L}$ may be the relevant confinement scale for the coaxial jet geometry. We plot on figure 13 the flapping frequency for the data of figures 6 and 7 as a function of the prediction of equation (1) with $L=H_{L}$ : we have only retained points for which $W e_{U i}>1$ and $M>5$, for which the inviscid confinement mechanism is expected to be relevant. The data is correctly aligned, in particular the series $H_{L}=20 \mathrm{~mm}-H_{G}=24 \mathrm{~mm}$ (symbol $\diamond)$ is aligned with the data for smaller geometries. In addition, equation (1) predicts frequency values to within $20 \%$ of experimental ones without any adjustable parameter.

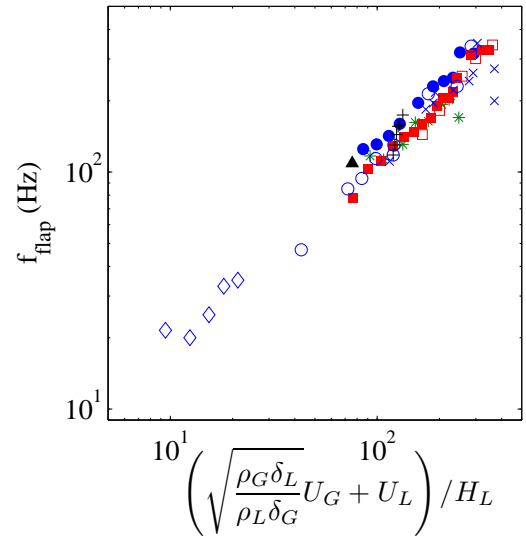

FIG. 13. Experimental flapping frequency as a function of the model of equation (1) for the subset of series $G_{1}$ for which the inviscid mechanism is expected to dominate $\left(W e_{U i}>1\right.$ and $M>5)$.

\section{GAS INDEPENDENT FLAPPING INSTABILITY}

Let us now discuss the frequency data corresponding to regime $G_{2}$. This regime corresponds to the series for which flapping frequency is independent of gas velocity on figure 6 . Figure 14 shows both the flapping and shear instability frequencies as a function of $U_{G}$ for the series $H_{L}=20 \mathrm{~mm}-H_{G}=5 \mathrm{~mm}$, fixed $U_{L}=0.28 \mathrm{~m} / \mathrm{s}$, pertaining to regime $G_{2}$. Clearly, both frequencies diverge beyond $U_{G}=50 \mathrm{~m} / \mathrm{s}$ : the shear instability frequency strongly increases with gas velocity while the flapping frequency remains roughly constant when $U_{G}$ is increased from 15 up to $140 \mathrm{~m} / \mathrm{s}$. Figure 15 illustrates the spatial development of the instability for the same conditions and $U_{G}=90 \mathrm{~m} / \mathrm{s}$ : the spectrum of interface position is dominated at close distance from the nozzle by a frequency of the order of $250 \mathrm{~Hz}$ (close to the shear instability frequency), but for $z>50 \mathrm{~mm}$ it exhibits a much smaller frequency, around $f \approx 35 \mathrm{~Hz}$ (close to the flapping frequency of figure 14). In this regime the flapping instability is therefore not controlled by the upstream shear instability.

In order to clarify what happens in this regime, we first describe the general mechanism which leads the liquid jet to flap. When a perturbation deforms the interface of the liquid jet, the fast gas flow is itself strongly perturbated and a wake is generated donwstream of the perturbation. Non-symmetric perturbations on the liquid then lead to strongly nonlinear non-symmetric perturbations in the gas stream, via air recirculation and lift-off behind waves. This mechanism has already been pointed out in the case of atomized liquid sheets by Lozano \& Barreras (2001) [28]. This is illustrated on figure 16, extracted from a previous study on a similar geometry, $H_{L}=8 \mathrm{~mm}$ and $H_{G}=1.7 \mathrm{~mm}$, for $U_{G}=10 \mathrm{~m} / \mathrm{s}$ and $U_{L}=0.4 \mathrm{~m} / \mathrm{s}$ [29]: the gas flow (indicated by longer white arrows) de- 


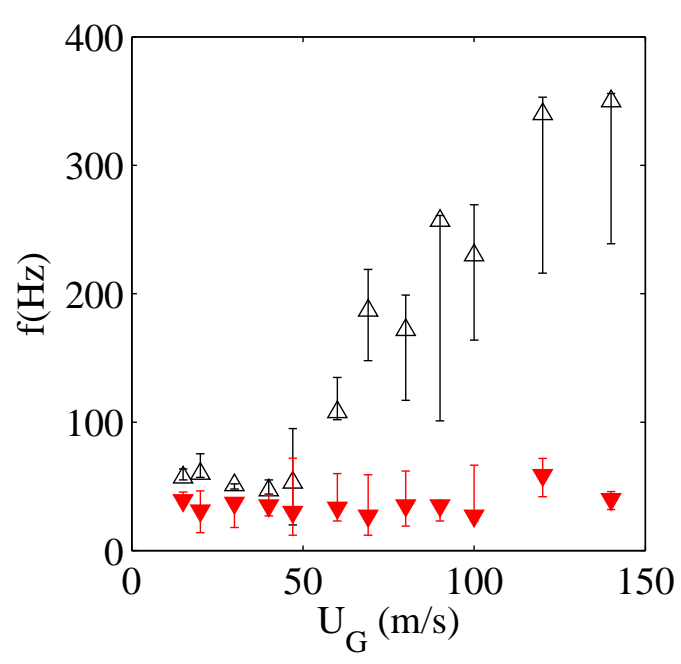

FIG. 14. Comparison between flapping frequency $(\boldsymbol{\nabla})$ and shear instability $(\triangle)$ frequency as a function of gas velocity for $H_{L}=20 \mathrm{~mm}, H_{G}=5 \mathrm{~mm}$ and fixed $U_{L}=0.28 \mathrm{~m} / \mathrm{s}$ : for $U_{G}>50 \mathrm{~m} / \mathrm{s}$ the flapping instability does not follow the shear instability and remains constant when $U_{G}$ is increased.

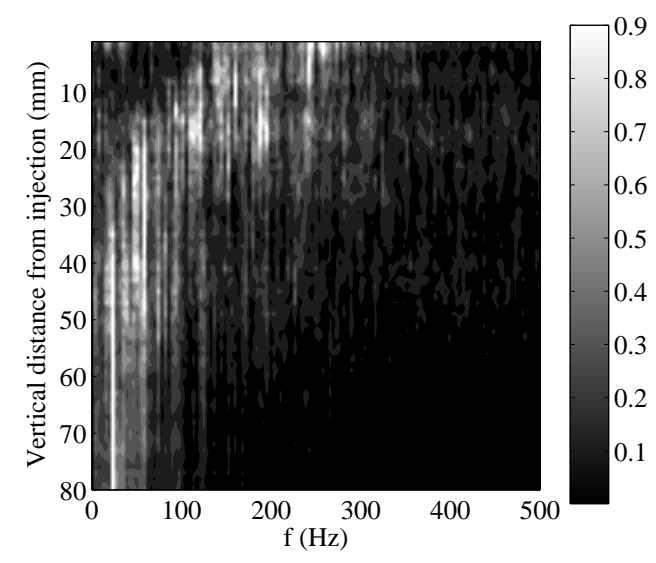

FIG. 15. Spatial variation of the interface position spectrum for $H_{L}=20 \mathrm{~mm} H_{G}=5 \mathrm{~mm} U_{L}=0.28 \mathrm{~m} / \mathrm{s}$ and $U_{G}=$ $90 \mathrm{~m} / \mathrm{s}$ : frequency decreases as one moves away from the nozzle.

taches after flowing past the liquid wave, and recirculates (white circular arrows). This is also illustrated in a high speed video deposited as suplemental material, for $H_{L}=5 \mathrm{~mm}, H_{G}=5 \mathrm{~mm}, U_{G}=15 \mathrm{~m} / \mathrm{s}$ and $U_{L}=0.22$ $\mathrm{m} / \mathrm{s}$ [30]: this video is taken at a frequency of $29 \mathrm{kHz}$ and exposure time $4 \mu \mathrm{s}$, and shows the liquid jet rotated of $90^{\circ}$ from its original vertical orientation. Gas flow is seeded with glycerine droplets (size $<5 \mu \mathrm{m}$ ), illuminated by an Argon laser slice in a plane containing the jet axis. Both the video and figure 16 show large recirculations behind non axisymmetric shear instability waves, which exert a couple on the liquid jet, and ultimately lead it to bend and deviate from its axis. This corresponds to

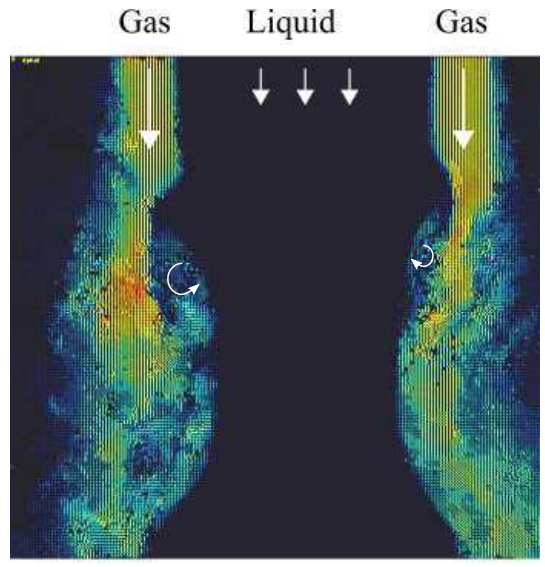

FIG. 16. PIV visualization of the velocity field in the air stream around an atomized water jet, $U_{G}=10 \mathrm{~m} / \mathrm{s}$ and $U_{L}=0.4 \mathrm{~m} / \mathrm{s}$, from Matas \& Cartellier (2013) [29]. Large recirculations appear downstream of the shear instability waves formed on the liquid surface. These recirculations will amplify the slight dissymmetry in the liquid jet by exerting a torque on the corresponding jet segment (see figure 11).

the sequence introduced above on figure 11. However, if shear waves are not spaced enough, i.e. when their wavelength is not large enough compared to the typical size $H_{L}$ of air recirculations, the previous mechanism is invalidated. We believe this is what happens for the data of series $G_{2}$. The data of figure 8 , where frequency in this regime increases with $U_{L}$, suggests that the relevant time scale for this regime must be built with $U_{L}$. Our experimental data suggest that length $H_{G}$ does not affect the frequency in this regime (compare for example series $\checkmark$ and $\boldsymbol{\nabla}$ in figure 6 ). We then choose to retain as the relevant length scale $H_{L}$, and build a Strouhal number $S t=f H_{L} / U_{L}$ to non dimensionalize flapping frequency. This Strouhal number is plotted against the Froude number $\mathrm{Fr}=U_{L} / \sqrt{g H_{L}}$ in figure 17. The data show that this Strouhal is constant and of order one for $\mathrm{Fr}>1$. For lower $F r, S t$ becomes much larger: this is due to the fact that as mentioned in section II B for the lowest $U_{L}$ investigated the relative increase in liquid velocity due to acceleration by gravity cannot be neglected over a distance of $H_{L}$. For the $U_{L}=0.08 \mathrm{~m} / \mathrm{s}$ and $H_{L}=15 \mathrm{~mm}$ series for example, after a free fall distance of $z=H_{L}$ liquid velocity reaches $0.55 \mathrm{~m} / \mathrm{s}$, i.e. almost seven times the liquid velocity at injection. More precisely, $U_{L}$ should be replaced by $U_{L}\left(1+2 F^{-2}\right)^{1 / 2}$. Similarly, the jet radius after a free fall distance of $H_{L}$ decreases due to mass conservation and becomes $H_{L}\left(1+2 F r^{-2}\right)^{-1 / 4}$. We plot on figure 17 (bottom) the variations of the modified Strouhal number $S t^{\prime}=S t\left(1+2 \mathrm{Fr}^{-2}\right)^{-3 / 4}$ taking into account these modified velocity and length: most of the data is gathered around $S t^{\prime} \approx 0.5$.

We finally discuss the issue of the boundary between regimes $G_{1}$ and $G_{2}$. The flapping instability mechanism is related with the gas recirculation and lift-off around asymmetric perturbations, and as mentioned above we 

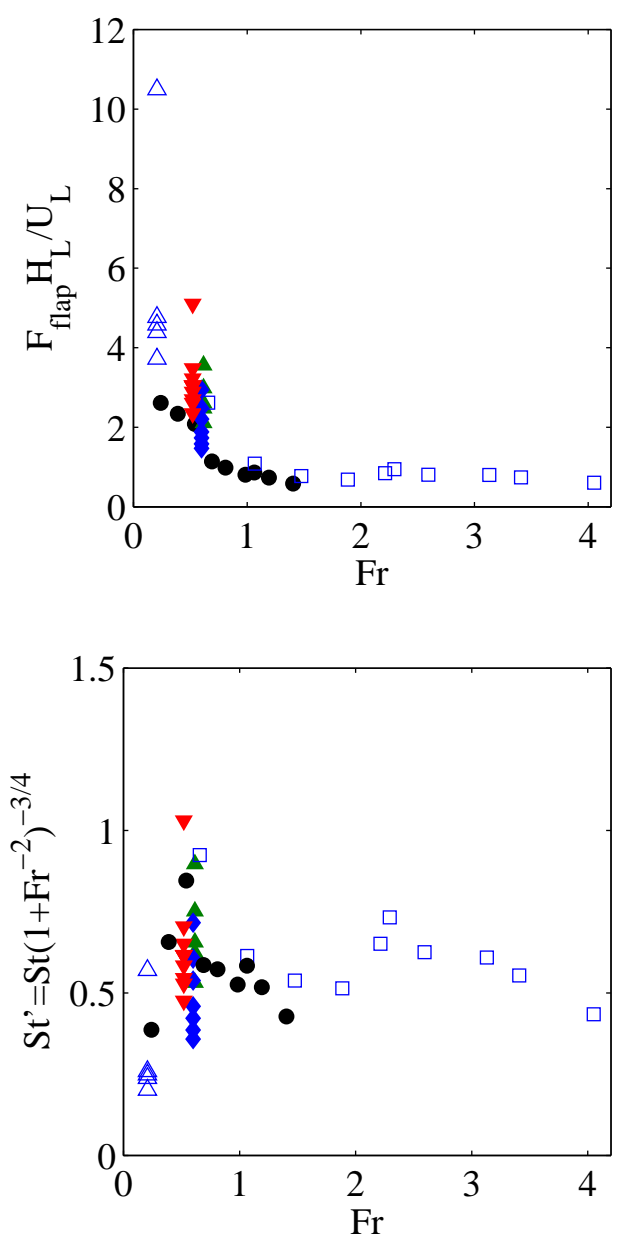

FIG. 17. Top: Variations of the Strouhal number $S t=$ $f H_{L} / U_{L}$ as a function of $F r$ for points in the $G_{2}$ regime, where flapping frequency is independent of gas velocity $U_{G}$. Same caption as in figures 6 and 8 ; Bottom: Same plot for Strouhal number $S t^{\prime}$ built with radius and velocity after a free fall of distance $H_{L}$.

believe the spacing between axial waves is a key parameter when examining the action of the gas on the jet. This spacing, namely the wavelength of the shear waves, is difficult to measure, in particular because of the strong spatial variations in velocity induced by the gas flow and gravity. Our strategy is to infer $\lambda$ from the shear waves frequency (measured via the spectra of radius variations, see section II) and velocity of the waves, which is expected to be close to $U_{c}=\left(\sqrt{\rho_{G}} U_{G}+\sqrt{\rho_{L}} U_{L}\right) /\left(\sqrt{\rho_{G}}+\right.$ $\left.\sqrt{\rho_{L}}\right) \approx \sqrt{\rho_{G} / \rho_{L}} U_{G}+U_{L}$ when the perturbation becomes non linear $[31,32]: \lambda=U_{c} / f_{\text {shear }}$. We then compute the ratio $H_{L} / \lambda$, which for a given geometry is a measure of the ability of a shear wave to generate a wake large enough to destabilize the liquid jet. Figure 18 left shows that the data labeled $G_{1}$, for which the flapping instability follows the shear instability, clearly corresponds to $H_{L} / \lambda<0.6$ : when the spacing between shear waves
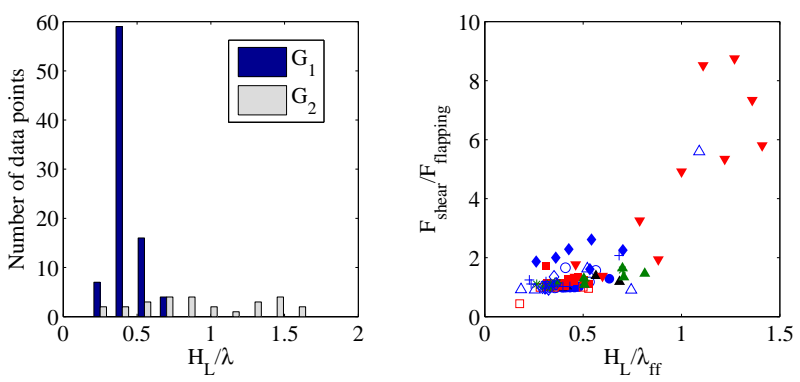

FIG. 18. Left: Histogram of $H_{L} / \lambda$ for all experimental points of figures 6 and 7: regime $G_{1}$ is observed for smaller $H_{L} / \lambda$ ; Right: ratio of shear frequency to flapping frequency as a function of $H_{L} / \lambda_{f f}$, for all data points of figures 6 and 7 (same legend).

is large enough, the flapping is synchronized with the shear instability. Conversely, most of the series belonging to the $G_{2}$ data, for which the flapping is not related to the shear instability, correspond to $H_{L} / \lambda>0.6$, i.e. to small wavelengths as argued previously. The estimate of the wavelength can be refined by considering as in figure 17 that it must be based on a free fall liquid velocity instead of $U_{L}$. Following this idea, figure 18 right represents for all the data points of figures 6 and 7 the ratio of the shear instability and flapping frequency, as a function of $H_{L} / \lambda_{f f}$. Here $\lambda_{f f}$ stands for the estimated wavelength accounting for free-fall velocity after a length $H_{L}: \lambda_{f f}=\left(\sqrt{\rho_{G} / \rho_{L}} U_{G}+U_{L} \sqrt{1+2 F r^{-2}}\right) / f_{\text {shear }}$. The largest $f_{\text {shear }} / f_{\text {flapping }}$ correspond as expected to the largest $H_{L} / \lambda_{f f}$ ratios, while both frequencies remain close to each other for larger wavelengths.

The first regime occurs when the wavelength associated with the shear instability is larger than the jet radius. Conversely, the second regime occurs when the shearinstability wavelength becomes too small compared with the jet radius: in this case the system prefers to amplify a larger scale, comparable with the jet size $H_{L}$. In that regime, the flapping arises from an opportunistic amplification of noisy perturbations and its response is no longer connected with the shear instability.

\section{CONCLUSION}

We have presented measurements of the frequency of the flapping instability, which leads an incompletely atomized liquid jet to exhibit oscillations on a scale large compared to its radius. By analyzing the variations of the flapping frequency over a large range of flow conditions and injector geometries, we have demonstrated the existence of two regimes: a first regime named $G_{1}$ where frequency increases with gas velocity, and a second regime named $G_{2}$ where frequency is independent of gas velocity. We have shown that in the first regime the flapping instability is triggered by the wake downstream of non axisymmetric modes of the shear instability. The flapping 
frequency in this regime directly depends on the mechanism which controls the shear instability, and which can be either confinement or a Yih mechanism cut off by surface tension. In this regime the flapping frequency is therefore itself a function of the parameters affecting these complex instabilities: liquid and gas velocity, but also geometry and vorticity thickness.

In regime $G_{2}$, the shear instability wavelength is too small compared with the jet radius for its wake to destabilize the jet. Hence, the system amplifies incoming perturbations with a resulting frequency of the order of $U_{L} / H_{L}$ which is no longer connected with the shear instability. At low Froude numbers this frequency must be corrected as $\left(U_{L} / H_{L}\right)\left(1+2 F r^{-2}\right)^{3 / 4}$ to account for the acceleration due to gravity. The transition between the two regimes is at first order controlled by the parameter $H_{L} / \lambda$. In this $G_{2}$ regime only $U_{L}$ and $H_{L}$ are expected to determine frequency, and other parameters (in particular $U_{G}$ and $\delta_{G}$ ) have no effect whatsoever on the instability.

In future work it would be interesting to determine if the above picture is also relevant for liquid sheet atomization, and in particular, if the second regime can be observed on liquid sheets in the limit of small shear instability wavelengths or equivalently of thick liquid sheets.

Finally, we have not discussed in this work the question of the size of the liquid fragments generated downstream. By stretching the liquid jet and redistributing liquid lumps, the flapping instability will evidently have a strong impact on the size and velocity distributions of the generated spray: the question of how this impacts the classical drop formation mechanisms will be the object of future work.

\section{ACKNOWLEDGMENTS}

The research leading to these results has received funding from the European Union Seventh Framework Programme (FP7/2007-2013) under grant agreement $\mathrm{n}^{\circ} 265848$ and was conducted within the FIRST project. The laboratory LEGI is part of the LabEx Tec 21 (Investissements d'Avenir - grant agreement $\mathrm{n}^{\circ} \mathrm{ANR}-11$ LABX-0030).

\section{APPENDIX: VISCOUS LINEAR STABILITY ANALYSIS}

The base flow profile is purely axial, and of the form introduced in [21], i.e. a sum of error functions, with a finite velocity at the interface mimicking the wake of the splitter plate.

$$
\left\{\begin{array}{r}
U(r)=U_{L 0} \operatorname{erf}\left(\frac{R-r}{\delta_{L}}\right)+U_{i}\left[\begin{array}{c}
\left.1+\operatorname{erf}\left(\frac{r-R}{\delta_{d} \delta_{L}}\right)\right] \\
\text { for } 0<r<R
\end{array}\right. \\
U(r)=\left[U_{G} \operatorname{erf}\left(\frac{r-R}{\delta_{G}}\right)+U_{i}\left[1-\operatorname{erf}\left(\frac{r-R}{\delta_{d} \delta_{L}}\right)\right]\right] \\
\times\left(\frac{1+\operatorname{erf}\left(\frac{H_{G}-r+R}{\delta_{G}}\right)}{2}\right) \\
\text { for } R<r<L_{G}
\end{array}\right.
$$

where $U_{L 0}$ is the liquid velocity far from the interface, $\delta_{L}$ the liquid vorticity thickness and $L_{G}$ is the radial distance at which a boundary condition with a solid wall is enforced, $L_{G}=10\left(R+H_{G}\right)$ for the present work. The contribution proportional to the interfacial velocity $U_{i}$ models the wake downstream the splitter plate, namely a vorticity layer of thickness $\delta_{d} \delta_{L}: \delta_{d}=1$ corresponds to the absence of a velocity deficit, while $\delta_{d} \ll 1$ corresponds to a near zero velocity at the interface [21]. The magnitude of the interfacial velocity $U_{i}$ is imposed by the continuity of tangential stresses:

$$
U_{i}=\frac{U_{G} \mu_{G} / \delta_{G}+U_{L} \mu_{L} / \delta_{L}}{\mu_{G}+\mu_{L}} \delta_{d} \delta_{L}
$$

where $\mu_{G}$ and $\mu_{L}$ are respectively the gas and liquid dynamic viscosities.

We next look at the stability of a small velocity perturbation $\mathbf{u}(r, \theta, z, t)$ superimposed on the above velocity profile. After linearization, we expand the perturbation on normal modes $\tilde{\mathbf{u}}(r, n, k, \omega) e^{i(n \theta+k z-\omega t)}$. We only look for axisymmetric perturbations, hence we take $n=0$. We then introduce the stream function $\phi$, related to the respectively axial and radial velocity components $\tilde{u}$ and $\tilde{v}$ with:

$$
\tilde{u}=\frac{1}{r} \frac{d \phi}{d r} \quad \tilde{v}=-\frac{i k}{r} \phi
$$

The equation for $\phi(r, k, \omega)$ is then a classical circular OrrSommerfeld equation:

$$
\begin{aligned}
& (U k-\omega)\left(\phi^{\prime \prime}-\frac{\phi^{\prime}}{r}-k^{2} \phi\right)+\phi k\left(\frac{U^{\prime}}{r}-U^{\prime \prime}\right)=-i \nu_{G / L} \\
& {\left[\phi^{\prime \prime \prime \prime}-\frac{2}{r} \phi^{\prime \prime \prime}+\frac{3}{r^{2}} \phi^{\prime \prime}-\frac{3}{r^{3}} \phi^{\prime}-2 k^{2}\left\{\phi^{\prime \prime}-\frac{\phi^{\prime}}{r}\right\}+k^{4} \phi\right]}
\end{aligned}
$$

where $\nu_{G / L}$ is the kinematic viscosity of the gas/liquid phase. We enforce boundary conditions at the outer wall: $\phi\left(L_{G}\right)=0$ and $\phi^{\prime}\left(L_{G}\right)=0$, as well as on the axis of symmetry of the system $\phi(0)=0$ and $\phi^{\prime}(0)=0$. Two solutions are then integrated in the gas phase from $r=L_{G}$ to $r=R$, and two solutions in the liquid phase from $r=0$ to $r=R$ (with Fortran 90). Continuity of respectively normal and tangential velocity and stress at the interface 
close the system:

i) $\phi_{L}=\phi_{G}$

ii) $\quad \phi_{G}^{\prime}-\phi_{L}^{\prime}=\frac{k \phi_{G}}{k U_{i}-\omega}\left(U_{G}^{\prime}(R)-U_{L}^{\prime}(R)\right)$

iii) $\mu_{G}\left(\phi_{G}^{\prime \prime \prime}-\frac{\phi_{G}^{\prime \prime}}{R}\right)-\phi_{G}^{\prime}\left[i \rho_{G}\left(k U_{i}-\omega\right)-\frac{\mu_{G}}{R^{2}}+3 \mu_{G} k^{2}\right]$

$+\phi_{G}\left(i k \rho_{G} U_{G}^{\prime}(R)+2 \mu_{G} \frac{k^{2}}{R}\right)+i \sigma \frac{k^{2}}{R^{2}} \frac{1}{k U_{i}-\omega}\left(1-k^{2} R^{2}\right)$

$=\mu_{L}\left(\phi_{L}^{\prime \prime \prime}-\frac{\phi_{L}^{\prime \prime}}{R}\right)-\phi_{L}^{\prime}\left[i \rho_{L}\left(k U_{i}-\omega\right)-\frac{\mu_{L}}{R^{2}}+3 \mu_{L} k^{2}\right]$

$+\phi_{L}\left(i \rho_{L} k U_{L}^{\prime}(R)+2 \mu_{L} \frac{k^{2}}{R}\right)$

iv) $\mu_{G}\left[k^{2} \phi_{G}+\frac{k U_{G}^{\prime \prime}(R)}{\omega-k U_{i}} \phi_{G}+\phi_{G}^{\prime \prime}-\frac{\phi_{G}^{\prime}}{R}\right]$

$$
=\mu_{L}\left[k^{2} \phi_{L}+\frac{k U_{L}^{\prime \prime}(R)}{\omega-k U_{i}} \phi_{L}+\phi_{L}^{\prime \prime}-\frac{\phi_{L}^{\prime}}{R}\right]
$$

As in Matas (2015) [23], we carry out a spatio temporal analysis: we solve for spatial branches, for a fixed complex $\omega_{i}=\mathcal{I} m(\omega)$. If when $\omega_{i}$ is decreased the shear branch pinches with either a confinement branch or a surface tension branch for $\omega_{i}>0$, then the instability is absolute and the corresponding value of $\omega_{i}$ is the absolute growth rate $\omega_{i 0}$. If no pinching occurs when $\omega_{i}$ has reached zero, then the instability is convective.

where $\sigma$ is the liquid/gas surface tension.

[1] A.H. Lefebvre, Atomization and Sprays (New York: Hemisphere, 1989).

[2] J. C. Lasheras and E. J. Hopfinger, "Liquid jet instability and atomization in a coaxial gas stream," Annu. Rev. Fluid Mech. 32, 275-308 (2000).

[3] P. Marmottant and E. Villermaux, "On spray formation," J. Fluid Mech. 498, 73 (2004).

[4] M. Hong, A. Cartellier, and E. J. Hopfinger, "Atomization and mixing in coaxial condition," in 4th International Conference on Launcher Technology Space Launcher Liquid Propulsion. Liège, Belgium (Belgium CNES Publ., 2002).

[5] C.M. Varga, J. C. Lasheras, and E. J. Hopfinger, "Initial breakup of a small-diameter liquid jet by a high-speed gas stream," J. Fluid Mech. 497, 405-434 (2003).

[6] Z. Farago and N. Chigier, "Morphological classification of disintegration of round liquid jets in a coaxial air stream," Atomization and Sprays 2, 137-153 (1992).

[7] H. Eroglu, N. Chigier, and Z. Farago, "Coaxial atomizer liquid intact lengths," Phys. Fluids 3, 303-308 (1991).

[8] C. Engelbert, Y. Hardalupas, and J.H. Whitelaw, "Breakup phenomena in coaxial air-blast atomizers," Proc. R. Soc. Lond. A , 189-229 (1995).

[9] W.O.H. Mayer and R. Branam, "Atomization characteristics on the surface of a round liquid jet," Experiments in Fluids 36, 528-539 (2004).

[10] M. Juniper and S.M. Candel, "The stability of ducted compound flows and consequences for the geometry of coaxial injectors," J. Fluid Mech. 482, 257-269 (2003).

[11] B. Leroux, O. Delabroy, and F. Lacas, "Experimental study of coaxial atomizers scaling. part i: dense core zone." Atomization and Sprays 17, 381-407 (2007).

[12] G. Charalampous, Y. Hardalupas, and A. Taylor, "Structure of the continuous liquid jet core during coaxial air-blast atomisation," Int. J. Spray and Combustion Dynamics 1 (2009).

[13] J. M. Locke, S. Pal, R. D. Woodward, and R. J. Santoro, "High Speed Visualization of LOX / GH 2 Rocket Injector Flowfield : Hot-Fire and Cold-Flow Experiments," in 46th AIAA/ASME/SAE/ASEE Joint Propulsion Conference \& Exhibit 25 - 28 July 2010, Nashville, TN AIAA 2010-7145, July (2010).

[14] A. Lozano, F. Barreras, C. Siegler, and D. Löw, "The ef- fects of sheet thickness on the oscillation of an air-blasted liquid sheet," Experiments in Fluids 39, 127-139 (2005).

[15] T. Arai and H. Hashimoto, "Disintegration of a thin liquid sheet in a cocurrent gas stream," in Proceedings of ICLASS'85, London, UK (1985) pp. VIB/1/1-VIB/1/7.

[16] A. Lozano, F. Barreras, G. Hauke, and C. Dopazo, "Longitudinal instabilities in an air-blasted liquid sheet," J. Fluid Mech. 437, 143-173 (2001).

[17] F. Couderc, Développement d'un code de calcul pour la simulation d'écoulements de fluides non miscibles. Application à la désintégration assistée d'un jet liquide par un courant gazeux., Ph.D. thesis, ENSAE Toulouse (2007).

[18] N. Odier, G. Balarac, C. Corre, and V. Moureau, "Numerical study of a flapping liquid sheet sheared by a highspeed stream," Int. J. Multiph. Flow 77, 196-208 (2017).

[19] J-P Matas, S Marty, and A Cartellier, "Experimental and analytical study of the shear instability of a gas-liquid mixing layer," Phys. Fluids 094112 (2011), $10.1063 / 1.3642640$.

[20] J.-P. Matas, A. Delon, and A. Cartellier, "Shear instability of an axisymmetric air-water coaxial jet," J. Fluid Mech. 843, 575 (2018).

[21] T. Otto, M. Rossi, and T. Boeck, "Viscous instability of a sheared liquid-gas interface : Dependence on fluid properties and basic velocity profile," Phys. Fluids 032103 (2013), 10.1063/1.4792311.

[22] D. Fuster, J.-P. Matas, S. Marty, S. Popinet, J. Hoepffner, A. Cartellier, and S. Zaleski, "Instability regimes in the primary breakup region of planar coflowing sheets," J. Fluid Mech. 736, 150-176 (2013).

[23] J.-P. Matas, "Inviscid versus viscous instability mechanism of an air-water mixing layer," J. Fluid Mech. 768, 375-387 (2015).

[24] P. A. M. Boomkamp and R. H. M. Miesen, "Classification of instabilities in parallel two-phase flow," Int. J. Multiph. Flow 22, 67-88 (1996).

[25] J.-P. Matas, M. Hong, and A. Cartellier, "Stability of a swirled liquid film entrained by a fast gas stream," Phys. Fluids 26 (2014).

[26] G. K. Batchelor and A. E. Gill, "Analysis of the stability of axisymmetric jets," J. Fluid Mech. 14, 529 (1962).

[27] J. J. Healey, "Enhancing the absolute instability of a boundary layer by adding a far-away plate," J. Fluid 
Mech. 579, 29 (2007).

[28] A. Lozano and F. Barreras, "Experimental study of the gas flow in an air-blasted liquid sheet," Experiments in Fluids 31, 367-376 (2001).

[29] J.-P. Matas and A. Cartellier, "Flapping instability of a liquid jet," Comptes Rendus de l'Académie des Sciences - Series IIB - Mechanics 341, 35-43 (2013).

[30] See Supplemental Material at [URL will be inserted by publisher], where these high-speed videos have been deposited.

[31] P. E. Dimotakis, "Two-dimensional shear-layer entrainment," AIAA J 24, 1791-1796 (1986).

[32] J. Hoepffner, R. Blumenthal, and S. Zaleski, "Selfsimilar wave produced by local perturbation of the kelvinhelmholtz shear-layer instability," Physical Review Letters 106, 104502 (2011). 